\title{
EFFICIENCY IS THE KEY
}

Dear Reader,

the $65^{\text {th }}$ IAA Commercial Vehicles will shortly be opening in Hannover and we are looking forward to seeing which solutions manufacturers and suppliers will be presenting to address the prevailing issues of fuel consumption, emissions reduction and driver assistance systems. One thing seems certain: electrification and autonomous driving, which are increasingly the focus of attention in passenger cars, are also playing a more and more important role in commercial vehicles.

For that reason, driver assistance and telematics are also likely to be among the dominant topics at the IAA Commercial Vehicles. The benefits of (semi-)autonomous driving for long-distance trucks are obvious. A truck that is connected with its environment via Vehicle-to-Vehicle (V2V) can sustainably improve transport efficiency. Mercedes-Benz presents such a connected system under the name Highway Pilot. The system shows that the technical requirements have been recognised and implemented. It is now a matter of adapting the legal framework.

Also important are questions such as how cooling systems can be made more efficient, not only to achieve better cooling performance but also to improve fuel efficiency. Mahle has developed such an energy-efficient dual-circuit cooling system, which is presented in this issue, and it will certainly be on show at the IAA. The company is promising a $1 \%$ reduction in fuel consumption. The use of steel instead of aluminium for pistons has the same objective.
Further measures to improve efficiency come from the field of aerodynamics for tractors and trailers, as well as a modular, electronically controlled air suspension system such as the one developed by Wabco. In this issue, the Hannover-based company presents a good overview of the current state of technology of modern trailer systems. These now feature electronically controlled safety and telematics systems. They still have huge potential for development in the future. But for the time being, efficiency is still the key.

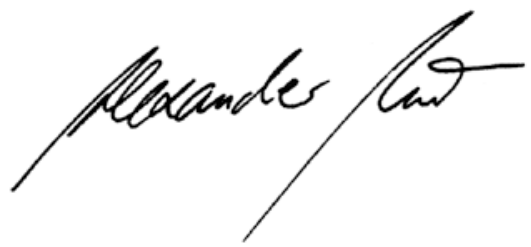

DR. ALEXANDER HEINTZEL, Editor in Chief Wiesbaden, 6 August 2014

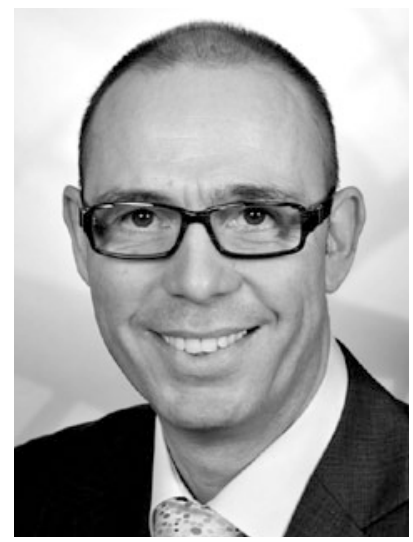

